\title{
Sobrecrecimiento bacteriano en trastornos funcionales del intestino
}

\author{
Ana María Madrid ${ }^{1}$, Carlos Defilippi $C^{1}$, Claudia Defilippi $^{1}$, \\ Jocelyn Slimming $A^{2}$, Rodrigo Q uera $\mathrm{P}^{1}$.
}

\section{Small intestinal bacterial overgrowth in patients with functional gastrointestinal diseases}

Background: Recent studies have described a high percentage of small intestinal bacterial overgrowth (SIBO) in patients with irritable bowel syndrome (IBS). However, the prevalence of SIBO has not been well established in other functional disorders. Aim: To evaluate the prevalence of SIBO in patients with different functional gastrointestinal disorders (FGID). Material and methods: Patients with FGID completed a self-administered questionnaire providing information to diagnose functional disorders on the basis of Rome II criteria. SIBO was assessed using a standardized lactulose breath test. A basal value of breath hydrogen (H2) $>20 \mathrm{ppm}$ and/or two lectures of $\mathrm{H} 2$ values $>20 \mathrm{ppm}$ during the first 60 minutes were considered suggestive of SIBO. Results: We studied 367 patients with a mean age of 50 years ( $87 \%$ females). Of these, 225 had IBS ( 45 constipation predominant, 121 diarrhea predominant and 59 alternating type), 33 had functional constipation, 83 had functional bloating and 26 had functional diarrhea. SIBO was found in $76 \%$ of patients with IBS, $73 \%$ of those with functional constipation, $69 \%$ of those with functional diarrhea and $68 \%$ of those with functional bloating. Conclusions: This study confirms a high percentage of SIBO in patients with IBS and other FGID. The eradication of SIBO should be considered as a therapeutic tool in these patients (Rev Méd Chile 2007; 135: 1245-52).

(Key w ords: Intestinal bacterial overgrowth; Irritable bowel syndrome; Lactulose tolerance test)

Recibido el 19 de junio, 2006. Aceptado el 7 de mayo, 2007.

${ }_{1}^{1}$ Departamento de Medicina, Sección Gastroenterología, Hospital Clínico de la Universidad de Chile. ${ }^{2}$ Servicio de Medicina, Sección de Gastroenterología, Hospital del Salvador. Santiago de Chile.

L os trastornos funcionales del tubo digestivo han sido definidos como una combinación de síntomas digestivos crónicos y recurrentes, en ausencia de patología orgánica. Un grupo de expertos ha establecido diversos subgrupos de-

Correspondencia a: Dra. Ana María Madrid S. Fono: 9788350. Fax: 9788349. E mail: amadrid@redclinicauchile.cl pendiendo del posible origen de los síntomas, de acuerdo a los denominados Criterios de Roma ${ }^{1}$.

El síndrome de intestino imitable (SII) definido de acuerdo a estos criterios, constituye uno de los cuadros clínicos más frecuentes en el mundo, es motivo de un elevado número de consultas a gastroenterólogos y médicos generales en Estados Unidos de Norteamérica y se acompaña de un deterioro de la calidad de vida de los pacientes ${ }^{2}$. En una encuesta realizada recientemente en la población 
de Santiago, se observó una incidencia de $22 \% 3$. La incidencia en otros países de la región no ha sido debidamente establecida 4 .

La sintomatología que permite formular este diagnóstico de acuerdo a los Criterios de Roma II es la presencia de dolor o malestar abdominal crónico, asociados a alteraciones del tránsito intestinal, cambios en el hábito defecatorio 0 ambos con una duración mínima de 12 semanas en los últimos 12 meses.

El origen de los síntomas es multifactorial. Se han descrito varios mecanismos fisiopatológicos: hiperalgesia visceral, alteraciones discretas de la motilidad intestinal y un inadecuado procesamiento de las aferencias viscerales a nivel central. Estas alteraciones estarían probablemente ligadas a factores genéticos y psicosociales ${ }^{5}$. Uno de los factores asociados descritos más recientemente es el sobrecrecimiento bacteriano intestinal (SBI) ${ }^{6}$.

Se estima que existe SBI en presencia de un número mayor a $10^{5}$ unidades formadoras de colonias (UFC)/mL de líquido intestinal, obtenido por aspiración. Sin embargo, debido a las limitaciones técnicas y baja sensibilidad de este méto$\mathrm{do}^{6}$, se ha utilizado en forma creciente el test de hidrógeno $\left(\mathrm{H}_{2}\right)$ en aire espirado en la detección del SBI, ya que su aplicación con criterios rigurosos, permite establecer esta condición en forma simple y no invasiva.

El SBI se ha descrito en presencia de alteraciones anatómicas del intestino delgado, como las observadas en el bypass yeyuno-ileal utilizado en el pasado para el tratamiento de la obesidad y en el intestino corto ${ }^{7-10}$. También se ha asociado a trastornos en la motilidad gastrointestinal, en especial del complejo motor migratorio $(\mathrm{CMM})^{11} \mathrm{y}$ frente a una disminución de la secreción gástrica de ácido clorhídrico ${ }^{12}$. En la mayoría de los pacientes esta condición produce síntomas, que incluyen alteraciones del hábito intestinal, principalmente diarrea, dolor abdominal, distensión y meteorismo, síntomas que son similares a los que presentan los pacientes con SII. En presencia de SBI es difícil establecer el origen de los síntomas.

Recientemente, Pimentel y col. han descrito SBI en un porcentaje elevado de pacientes con SII mediante el test de $\mathrm{H}_{2}$ espirado con lactulosa ${ }^{13}$. Este hallazgo y su rol en la génesis de los síntomas y los efectos de su erradicación han sido motivo de controversia ${ }^{14}$.
En la literatura no está claramente establecida la prevalencia de SBI en otras patologías funcionales establecidas por los Criterios de Roma II que no corresponden al SII.

Los objetivos de nuestro trabajo fueron confirmar y establecer la prevalencia de SBI en pacientes con SII en nuestro medio, en sus diferentes presentaciones clínicas y, además, determinar su presencia y distribución porcentual en otros subgrupos de los denominados trastornos funcionales intestinales.

\section{PACIENTES Y MÉTODO}

Se estudiaron en forma prospectiva pacientes ambulatorios derivados al Laboratorio de Enfermedades Funcionales Digestivas de nuestro hospital, para investigar la posible presencia de SBI mediante test de $\mathrm{H}_{2}$ en aire espirado como causa de sus síntomas digestivos. Entre enero de 2003 y octubre de 2004, se estudiaron 367 pacientes que cumplían criterios para patología digestiva funcional y en los que se descartó organicidad mediante una ecotomografía abdominal, endoscopia digestiva alta o colonoscopia. No se incluyeron pacientes con antecedentes de cirugía del tubo digestivo, diabetes mellitus, neoplasias o que no cumplían con los criterios de cronicidad para patología funcional.

Todos los enfermos contestaron la encuesta de Roma II para categorización de trastornos funcionales digestivos en su versión en español, que incluye los siguientes ítems: malestar o dolor abdominal, su localización, intensidad, asociación con trastornos del hábito defecatorio, frecuencia de las evacuaciones, consistencia de las deposiciones, presencia de sangre o mucosidades en las deposiciones, sensación de alivio después de evacuar, sensación de evacuación incompleta, náuseas, vómitos, meteorismo y distensión abdominal. Se diagnosticó SII en sujetos que presentaron malestar o dolor abdominal, difuso o en el hemiabdomen inferior, acompañado de meteorismo, constipación o diarrea u otras alteraciones de la evacuación, siendo el síntoma predominante el dolor, durante los últimos 12 meses, por un periodo mínimo de 12 semanas. A su vez, los pacientes con SII se subclasificaron según Roma II en las categorías de: predominio constipación, 
predominio de diarrea y alternantes, dependiendo de la calidad y frecuencia de las deposiciones ${ }^{1}$.

Se clasificaron como diarrea funcional (DF) los sujetos con una frecuencia defecatoria de 30 más evacuaciones por día en más de $75 \%$ del tiempo, sin dolor abdominal, en al menos 12 semanas, no necesariamente consecutivas en los últimos 12 meses. Constipación funcional (CF) los sujetos con una frecuencia defecatoria menor a 3 evacuaciones por semana, en ausencia de dolor 0 malestar abdominal o cuando éste se presentó con carácter leve en al menos 12 semanas en los últimos 12 meses y se consideró meteorismo funcional (MF) su aparición como síntoma principal no asociado a alteraciones del tránsito o de la evacuación, también en al menos 12 semanas en los últimos 12 meses. Esta encuesta permite excluir otras categorías de trastornos funcionales, como la dispepsia funcional y el dolor abdominal funcional.

El SBI se investigó mediante test de $\mathrm{H}_{2}$ en aire espirado con lactulosa. Previo ayuno de $12 \mathrm{~h}$, se tomaron muestras de aire cada 10 min durante 180 min al término de una espiración en una jeringa antes y después de ingerir $25 \mathrm{~g}$ de lactulosa. Las muestras fueron analizadas en un cromatógrafo de gases (Quintron MicroLyzer CM2 USA) y los resultados se expresaron en partes por millón (ppm). Se consideró SBI la presencia de un valor basal de hidrógeno sobre $20 \mathrm{ppm}$ o 2 lecturas sobre $20 \mathrm{ppm}$ durante los primeros $60 \mathrm{~min}$. La cuantificación del SBI se realizó sumando las lecturas del $\mathrm{H}_{2}$ espirado durante los primeros 60 min $\left(\mathrm{H}_{2} 60\right)$. Además, se evaluó el tiempo de tránsito orocecal (TTOC), que corresponde al tiempo transcurrido entre la ingesta de la lactulosa y el aumento de $\mathrm{H}_{2}$ espirado causado por la fermentación de la flora bacteriana normal colónica (normal 80-100 min). En caso de existir SBI se observan dos elevaciones, una temprana que ocurre por la degradación de la lactulosa por las bacterias en el intestino delgado y la segunda a nivel del colon.

Estadística. Para comparar la presencia de SBI entre los distintos grupos se utilizó test de Chi cuadrado y Fisher exacto. En el análisis de la cuantificación del SBI se usó Anova para grupos no pareados.

\section{RESULTADOS}

La encuesta permitió seleccionar 367 pacientes con patología digestiva funcional y establecer 4 subgrupos: 225 pacientes con SII, $83 \mathrm{MF}, 33$ pacientes con CF y 26 pacientes con DF. La Figura 1 muestra su distribución porcentual.

Entre los subgrupos de pacientes con SII, el subgrupo con diarrea fue el más numeroso y no se observaron diferencias en edad y distribución por sexo (Tabla 1). En los grupos de meteorismo funcional, diarrea y constipación funcional tampoco se observaron diferencias en la distribución por sexo y edad (Tabla 2).

Todos los grupos de patologías funcionales presentaron una mayor prevalencia de mujeres ( $\mathrm{p}$ $<0,005$ ), siendo ésta aún mayor en el grupo con constipación funcional (Figura 2).

La gran mayoría de los pacientes con SII estudiados presentaron SBI: 76,4\% (rango 73\%$80 \%)$ (Tabla 3). En los grupos funcionales no SII la frecuencia de SBI también fue elevada y sin diferencias con los pacientes con SII (Tabla 4).

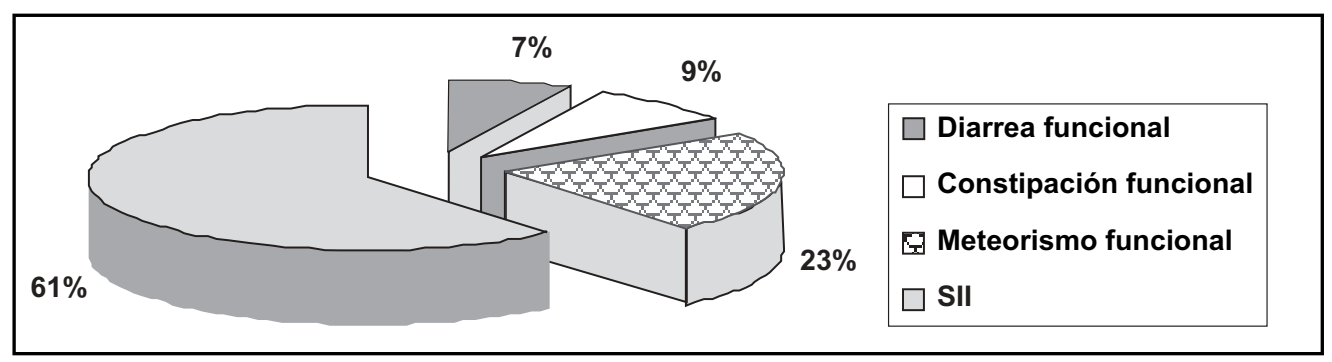

Figura 1. Distribución porcentual de los distintos subgrupos. 
Tabla 1. D istribución por sexo y edad de los pacientes con SII

\begin{tabular}{|lccccccc|}
\hline $\begin{array}{l}\text { Pacientes con síndrome } \\
\text { de intestino irritable } \\
\text { (subtipos) }\end{array}$ & $\mathrm{n}$ & \multicolumn{2}{c}{ Promedio } & \multicolumn{2}{c}{ Mujeres } & \multicolumn{2}{c|}{ Hombres } \\
& & & $\begin{array}{l}\text { Rango } \\
\text { (años) }\end{array}$ & $\mathrm{n}$ & $\%$ & $\mathrm{n}$ & $\%$ \\
Alternante & 59 & 43 & $(15-78)$ & 41 & 69,5 & 18 & 31,5 \\
Diarrea & 121 & 46 & $(15-78)$ & 85 & 70 & 36 & 30,0 \\
Constipación & 45 & 44 & $(16-75)$ & 38 & 84,4 & 7 & 15,6 \\
SII total & 225 & 45,5 & $(15-78)$ & 164 & 72,8 & 61 & 27,2 \\
\hline
\end{tabular}

Tabla 2. Características de los grupos con patología funcional no SII

\begin{tabular}{|lccccccc|}
\hline Subgrupo funcional & $\mathrm{n}$ & $\overline{\mathrm{x}}$ edad & Rango & \multicolumn{2}{c|}{ Mujeres } & \multicolumn{2}{c|}{ Hombres } \\
& & & (años) & $\mathrm{n}$ & $\%$ & $\mathrm{n}$ & $\%$ \\
\hline Meteorismo & 85 & 42 & $(15-79)$ & 59 & 71 & 26 & 29,0 \\
Diarrea & 26 & 49 & $(18-79)$ & 21 & 80,8 & 5 & 19,2 \\
Constipación & 33 & 41 & $(18-73)$ & 32 & 97 & 1 & 3,0 \\
\hline
\end{tabular}

Tabla 3. Porcentaje de SBI en los diferentes subgrupos de SII

\begin{tabular}{|c|c|c|c|c|c|c|c|}
\hline \multirow{2}{*}{$\begin{array}{l}\text { Pacientes con síndrome } \\
\text { de intestino irritable }\end{array}$} & \multirow[t]{2}{*}{$\mathrm{n}$} & \multicolumn{2}{|c|}{ SBI } & \multicolumn{2}{|c|}{ SBI/O } & \multicolumn{2}{|c|}{$\mathrm{SBI} / 0^{7}$} \\
\hline & & $\mathrm{n}$ & $\%$ & $\mathrm{n}$ & $\%$ & $\mathrm{n}$ & $\%$ \\
\hline Constipación & 45 & 33 & 73,3 & 31 & 81,6 & 2 & 28,5 \\
\hline Diarrea & 121 & 92 & 76,0 & 63 & 74,1 & 29 & 80,5 \\
\hline Alternantes & 59 & 47 & 79,7 & 33 & 80,5 & 14 & 77,8 \\
\hline SII total & 225 & 172 & 76,4 & 127 & 77,4 & 45 & 62,3 \\
\hline
\end{tabular}

Tabla 4. Porcentaje de SBI en los diferentes subgrupos de patología funcional digestiva no SII

\begin{tabular}{|lcccccrr|}
\hline Subgrupo funcional & $\mathrm{n}$ & \multicolumn{2}{c}{$\mathrm{SBI}$} & \multicolumn{2}{c}{ SBI/o } & \multicolumn{2}{c|}{ SBI/ơ } \\
& & $\mathrm{n}$ & $\%$ & $\mathrm{n}$ & $\%$ & $\mathrm{n}$ & $\%$ \\
\hline Meteorismo & 83 & 56 & 67,5 & 40 & 67,8 & 16 & 61,5 \\
Diarrea & 26 & 18 & 69,2 & 13 & 61,9 & 5 & 100 \\
Constipación & 33 & 24 & 72,7 & 23 & 71,9 & 1 & 100 \\
\hline
\end{tabular}

Sólo se observó mayor porcentaje de SBI en el grupo SII alternante versus el grupo con meteorismo funcional ( $p<0,005)$.
Tampoco se observaron diferencias en la magnitud de SBI $\left(\mathrm{H}_{2} 60\right)$ entre los diferentes grupos de patología funcional estudiados (Figura 3). 


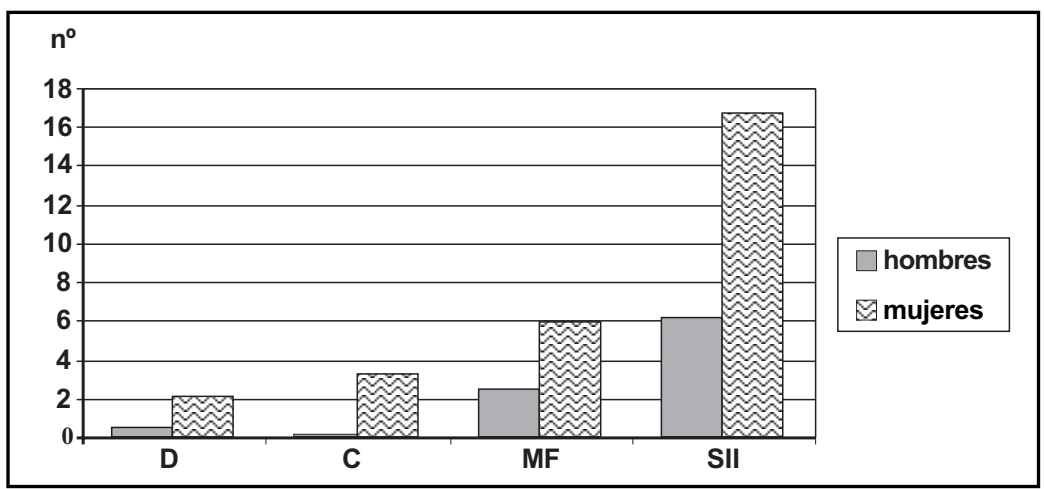

Figura 2. Distribución por sexo según subgrupos.

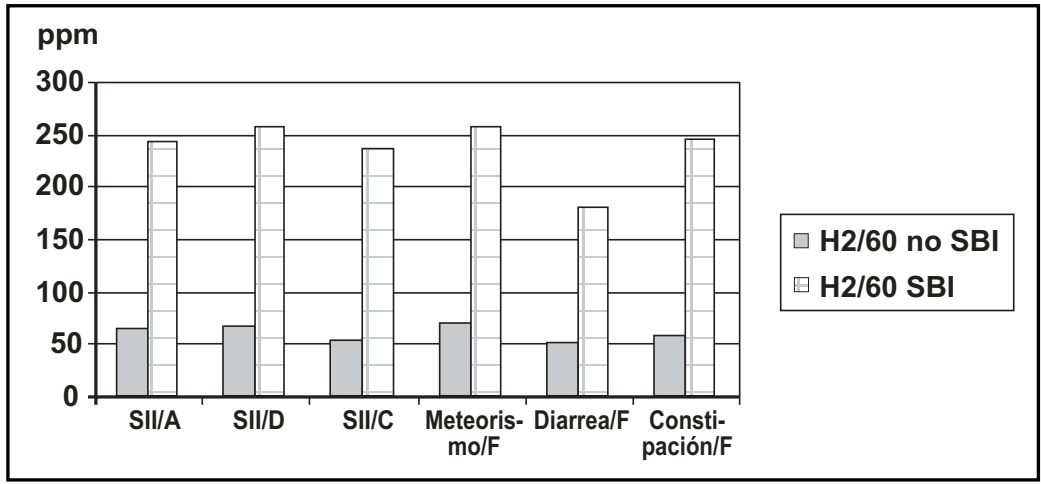

Figura 3. Cuantificación del sobrecrecimiento bacteriano intestinal, en los distintos subgrupos.

\section{DISCUSIÓN}

En el presente trabajo se comparó la presencia de SBI en pacientes con diferentes subgrupos de trastornos funcionales intestinales, seleccionados de acuerdo a los Criterios de Roma II. El SBI intestinal estuvo presente en un porcentaje elevado en todos los grupos estudiados y la incidencia en la mayoría de los subgrupos no fue diferente.

Nuestra observación es coincidente con la de Pimentel y col, quienes demostraron la presencia del SBI en $78 \%$ de los pacientes con SII; estos autores, además, demostraron que la erradicación de SBI fue seguida de una reducción de los síntomas $^{13}$. Un aumento de SBI en pacientes con SII comparados con un grupo control, fue también observado en otro lugar geográfico ${ }^{15}$. Sin embargo, estos hallazgos no han sido confirmados por otros autores ${ }^{16,17}$. Estas diferencias pudieran estar más bien relacionadas con la técnica utilizada en la detección de SBI o en la interpretación de los resultados ${ }^{14}$.

Nuestros hallazgos y los observados en la literatura plantean varias interrogantes en relación al rol que juega el SBI en los trastornos funcionales del tubo digestivo.

La presencia de SBI se ha observado en población asintomática, pero en un porcentaje mucho menor que en los pacientes funcionales ${ }^{15}$. Una observación similar se ha efectuado en nuestro medio ${ }^{18}$, lo cual descarta que estemos en presencia de un fenómeno generalizado.

En ausencia de alteraciones anatómicas o bioquímicas, el mecanismo más frecuentemente invocado para explicar la presencia de SBI es un trastorno de la motilidad intestinal ${ }^{11,19,20}$. En el 
caso de SII, se han descrito alteraciones motoras del intestino delgado, sin embargo, los resultados observados han sido motivo de controversia ${ }^{21-24}$. Recientemente se han descrito alteraciones del complejo motor migratorio en pacientes con SII y $\mathrm{SBI}^{25}$, sin embargo, este hallazgo es de difícil interpretación, ya que la erradicación del SBI mejoró a lo menos parcialmente el trastorno motor.

El o los posibles mecanismos a través de los cuales un aumento de la población bacteriana pudiera dar origen a las manifestaciones clínicas propias del SII u otras patologías funcionales del tubo digestivo, han sido sugeridas por diferentes estudios, entre ellos: a) un aumento de la producción de gas al interactuar las bacterias con los nutrientes, a lo cual se sumaría una alterada distribución del gas a lo largo del intestino ${ }^{26}$; b) al inducir alteraciones de la motilidad del intestino delgado, mencionadas anteriormente; c) a través de alteraciones del metabolismo de la serotonina ${ }^{27}$; d) a través de la producción de citoquinas, asociada a una disregulación entre citoquinas antiinflamatorias y proinflamatorias determinada por factores genéticos ${ }^{28-30}$.

Sin embargo, llama la atención que pacientes con tan diferentes sintomatologías como constipación y diarrea y en presencia o no de síntomas abdominales presenten SBI en la misma proporción.

El mecanismo por el cual pacientes con SBI podrían presentar constipación o diarrea como síntoma predominante, ha sido en parte dilucidado por algunas observaciones que han mostrado que en pacientes con SII la presencia en forma exclusiva de una flora bacteriana productora de metano, se asocia invariablemente con constipación. En cambio, frente a una flora productora de $\mathrm{H}_{2} \mathrm{O}$ mixta puede observarse aparición tanto de diarrea como de constipación ${ }^{31}$. La importancia del rol de la flora bacteriana productora de

\section{REFERENCIAS}

1. Drossman DA. The functional gastrointestinal disorders and the Rome II process, en Drossman DA The functional Gastrointestinal Disorders, Degnon Ass McLean, VA U.S.A. 2000; 1-29. metano como causa de constipación en pacientes con SII, ha sido sugerida por estudios que revelan que al tratar a estos pacientes con neomicina se observa una mejoría significativa de la constipación $^{32}$. Otros estudios han demostrado que la rifaximina, un antibiótico no absorbible, es superior que el placebo al evaluar el porcentaje de pacientes que responden y la mejoría global clínica ${ }^{33}$.

Este aspecto no fue explorado en nuestro estudio, ya que no contamos con la medición de metano. Una hipótesis muy especulativa podría hacer plantear que una discreta alteración de la motilidad intestinal en los diferentes grupos estudiados pudiera favorecer la aparición de SBI. El tipo de flora metanogénica o productora de $\mathrm{H}_{2}$ pudiera a su vez, determinar el tipo de tránsito intestinal predominante y una reacción del huésped ante el aumento de la flora bacteriana en la parte alta del tubo digestivo, pudiera establecer las diferencias en la aparición de dolor o de otros síntomas. En relación con este aspecto algunos trabajos han sugerido un probable rol de carácter genético en los trastornos funcionales digesti$\operatorname{vos}^{34}$.

A pesar de la controversia analizada, estimamos que el factor SBI debe ser considerado como una posibilidad terapéutica mediante el uso de antibióticos, en especial en aquellos pacientes que presentan síntomas refractarios a la terapia habitual. Estudios idealmente controlados y randomizados, permitirían evaluar adecuadamente esta opción de tratamiento.

\section{Agradecimientos}

Agradecimientos al Sr. José Matus Figueroa, asistente del Laboratorio de Enfermedades Funcionales Digestivas de nuestro Hospital Clínico, por su valiosa colaboración en la realización de los exámenes de test de $\mathrm{H}_{2}$.

2. Andrews EB, Eaton SC, Hows KA, Hopkins JS, Ameen V, Hamm LR et al. Prevalence and demographics of irritable bowel syndrome: results from a large web-based survey. Aliment Pharmacol Ther 2005; 22: 935-42.

3. Madrid AM, Olguín F, Larraín S, Martínez N, Cortés 
J, Quera R et al. Prevalencia del síndrome de intestino irritable en población chilena: estudio preliminar. Gastr Latinoam 2005; 16: 392 [Abstract].

4. Valenzuela J, Alvarado J, Cohen H, Damiao A, Francisconi C, Frugona L et al. Un consenso latinoamericano sobre el síndrome del intestino irritable. Gastroenterol Hepatol 2004; 27: 325-43.

5. Drossman DA, Camileri M, Mayer E, Whitehead W. AGA technical review on irritable bowel syndrome. Gastroenterology 2002; 123: 2108-31.

6. LiN HC. Small intestinal bacterial overgrowth. A framework for understanding irritable bowel syndrome. JAMA 2004; 292: 852-8.

7. Leung FW, Drenic EJ, Stanley TM. Intestinal complication involving the excluded small bowel segment. Am J Gastroenterol 1982; 77: 67-77.

8. Passaro E, Drenice, WiLson SE. Bypass enterites. A new complication of jejunoileal bypass for obesity. Am J Surg 1976; 131: 169-74.

9. Justino SR, Gonçalves MC, Maculevicius J, De Morais MB, Sing TCH, Halpern A et al. Fasting breath hydrogen concentration in short bowel syndrome patients with colon incontinuity before and after antibiotic therapy. Nutrition 2004; 20 : 187-91.

10. Quera R, Quigley E, Madrid AM. Sobrecrecimiento bacteriano intestinal. Rev Méd Chile 2005; 133: 1361-70.

11. Vatrappen C, Janssens J, Hellemans J, Goohoos Y. The interdigestive motor complex of normal subjects and patients with bacterial overgrowth of the small intestine. J Clin Invest 1977; 59: 1158-66.

12. Saltzman JR, Kowdley KV, Pedrasa MC, Sepe T, Golner B, Perrone G et al. Bacterial overgrowth without clinical malabsorption in elderly hypochlorhydric subjects. Gastroenterology 1994; 106 : 615-23.

13. Pimentel M, Chow EJ, Lin H. Eradication of small intestinal bacterial overgrowth reduces symptoms of irritable bowel syndrome. Am J Gastroenterol 2000; 95: 3503-6.

14. SimRen M, Stotzer PO. Use and abuse of hydrogen breath tests. Gut 2006; 55: 297-303.

15. Lupascu A, Gabrieu M, Lauritano E, Scarpeuni E, Santoliquido A, Cammarota G et al. Hydrogen glucose breath test to detect small intestinal bacterial overgrowth: A prevalence case-control study in irritable bowel syndrome. [Abstract] Gastroenterology 2005; 128: 1742.
16. Posserud I, Ringström G, Stolzer PO, Bjornsson E, ABRAHAMSSON H, SimRen M. Small intestinal bacterial overgrowth (SIBO) in irritable bowel syndrome (IBS). [Abstract] Neurogastroenteral Motil 2004; 16: 839 .

17. WaLter B, VANNeR SJ. Detection of bacterial overgrowth in IBS using the lactulose $\mathrm{H}$ breath test: comparison with C-D-Xylose and healty controls. Am J Gastroenterol 2005; 100: 1566-70.

18. Planzer M, Madrid AM, Quera R, Defilppi CL, DefiLpi C, Hurtado C. Tiempo de tránsito orocecal: influencia del sexo, la edad y el sobrecrecimiento bacteriano. [Resumen] Gastr Latinoam 2002; 13: 376.

19. Stotzer PO, Björnsson ES, Abrahamsson H. Interdigestive and postprandial motility in small-intestinal bacterial overgrowth. Scand J Gastroenterol 1996; 31: 875-80.

20. Nieuwenhuijs VB, Verheem A, Van DuijvenbodeBeumer H, Visser MR, Verhoef J, Gooszen HG, AKKERMANS LMA. The role of interdigestive small bowel motility in the regulation of gut microflora, bacterial overgrowth, and bacterial translocation in rats. Ann Surg 1998; 228: 188-93.

21. Malageiada J-R, Stanghewni V. Manometric evaluation of functional upper gut symptoms. Gastroenterology 1985; 88: 1223-31.

22. KeLOW JE, PhiшPS SF, MiLer LJ, Zinsmeister AR. Dysmotility of the small intestine in irritable bowel syndrome. Gut 1988; 29: 1236-43.

23. Kingham JGC, Bown R, Colson R, Ciark ML. Jejunal motility in patients with functional abdominal pain. Gut 1984; 25: 375-80.

24. Defluppi C, Chesta J, DefilpPi CL. Relación entre síntomas funcionales del aparato digestivo $\mathrm{y}$ motilidad del intestino delgado. Rev Méd Chile 1990; 118: 1201-5.

25. Pimentel M, Soffer EE, Chow EJ, Kong Y, Lin HC. Lower frequency of MMC is found in IBS subjects with abnormal lactulose breath test, suggesting bacterial overgrowth. Dig Dis Sci 2002; 47: 263943.

26. Harder H, Serra J, Azpiroz F, Passos MC, Aguadé S, Malageiada J-R. Intestinal gas distribution determines abdominal symptoms. Gut 2003; 52: 170813.

27. Pimentel M, Kong Y, Park S. IBS subjects with methane on lactulose breath test have lower postprandial serotonin levels than subjects with hydrogen. Dig Dis Sci 2004; 49: 84-7. 
28. Cowns SM. Dysregulation of peripheral cytokine production in irritable bowel syndrome. Am J Gastroenterol 2005; 100: 2517-18.

29. Van Der Week PP, Van De Berg M, De Kroon Ye, Verspaget HW, Masclee AA. Role of tumor necrosis factor-alpha and interleukin-10 gene polymorphisms in irritable bowel syndrome. Am J Gastroenterol 2005; 100: 2510-16.

30. Gonsalkorale WM, Perrey C, Pravica V, Whorwell PJ, Hutchinson IV. Interleukin 10 genotypes in irritable bowel syndrome: evidence for an inflammatory component? Gut 2003; 52: 91-3.

31. Pimentel M, Mayer AG, Park S, Chow Ej, Hasan A, Kong Y. Methane production during lactulose breath test is associated with gastrointestinal disease presentation. Dig Dis Sci 2003; 48: 86-92.
32. Pimentel M, Chatterjee S, Chow EJ, Park S, Kong Y. Neomycin improves constipation-predominant irritable bowel syndrome in a fashion that is dependent on the presence of methane gas: subanalysis of a double-blind randomized controlled study. Dig Dis Sci 2006; 51: 1297-301.

33. Pimentel M, Park S, Mirocha J, Kane SV, Kong Y. The effect of a nonabsorbed oral antibiotic (rifaximin) on the symptoms of the irritable bowel syndrome: a randomized trial. Ann Intern Med 2006; 145: 557-63.

34. ParK MI, CAMLLeRI M. Genetics and genotypes in irritable bowel syndrome: implications for diagnosis and treatment. Gastroenterol Clin North Am 2005; 34: 305-17. 